\title{
Le spectromètre de masse à temps de vol DEPIL et son utilisation pour la mesure de masses de protéines élevées dépassant 5000 u $\left(^{*}\right)$ : insuline et lyzozyme
}

\author{
S. Della-Negra, C. Deprun et Y. Le Beyec \\ Institut de Physique Nucléaire, B.P. no 1, F-91406 Orsay Cedex, France
}

(Reçu le 6 décembre 1985, révisé le 7 mars 1986, accepté le 11 mars 1986)

\begin{abstract}
Résumé. - Un spectromètre de masse à temps de vol a été construit à l'Institut de Physique Nucléaire d'Orsay pour mesurer des masses de molécules diverses. La gamme de masses accessibles est comprise entre 1 et $20000 \mathrm{u}$. Nous présentons ici une description succincte de l'appareillage avec des exemples de mesures de hautes masses.
\end{abstract}

\begin{abstract}
A new time-of-flight mass spectrometer has been built at the Institut to measure high mass molecules. The mass range is between 1 and $20000 \mathrm{u}$. We discuss briefly the apparatus and give results on high mass measurements.
\end{abstract}

\section{Introduction.}

Les mesures de temps de vol " événement par événement " ont bénéficié dans les années passées de développements techniques importants, à la fois au niveau de l'électronique rapide et au niveau des phénomènes à détecter (rayonnements laser, U.V., gamma, R.X., ...) ou impact de particules $(\beta$ et $\alpha$, noyaux d'énergies diverses ou fragment de noyaux, atomes ou agrégats atomiques et moléculaires...). Après avoir mis au point et utilisé à l'IPN d'Orsay un appareil d'identification de masse par temps de vol des noyaux radioactifs (émetteurs $\alpha$ et $\beta$ ) produits au cours de réactions nucléaires par ions lourds [1, 2], nous nous sommes intéressés à l'extension à d'autres domaines de ce type de technique [3]. En effet, la spectrométrie de masse par temps de vol pour des composés divers connaît depuis peu un essor grandissant du fait des améliorations sur les mesures de temps, mais également grâce aux méthodes nouvelles de désorption-ionisation qui sont bien adaptées à ces mesures de temps. Les méthodes les plus fréquemment utilisées sont l'impact laser de courte durée (quelques ns) [4, 5], des faisceaux d'ions pulsés de quelques $\mathrm{keV}[6,7]$, des ions de plusieurs $\mathrm{MeV}$ provenant d'un accélérateur ou plus simplement d'une source radioactive par fission spontanée du ${ }^{252} \mathrm{Cf}$. Dans cette dernière méthode, les ions de quelques $\mathrm{MeV}$ sont détectés individuellement.

$\left(^{*}\right) \mathrm{u}$ : unité de masse atomique.
L'utilisation du ${ }^{252} \mathrm{Cf}$ proposé par R. Macfarlane et al. [8], s'est répandue dans quelques laboratoires [9] et s'est révélée être particulièrement efficace pour la désorption de très grosses molécules [10-12]. Des développements instrumentaux originaux de cette méthode ont été entrepris à l'IPN. En particulier un nouveau spectromètre à temps de vol a été réalisé $\left({ }^{1}\right)$. Nous présentons ici des résultats sur la mesure de masse de grosses molécules obtenus avec cet appareil (masses moléculaires supérieures à $5000 \mathrm{u}$ ).

\section{Méthode expérimentale.}

1.1 GÉNÉRALITÉS : DESCRIPTION DE L'APPAREIL. Le principe de la méthode a déjà été décrit [3]. La figure 1 montre schématiquement la configuration adoptée pour le nouvel appareil DEPIL (DEsorption Par Ions Lourds). La longueur de temps de vol notée $L$ sur la figure est variable de $12 \mathrm{~cm}$ à $95 \mathrm{~cm}$. Cette possibilité d'une longueur variable est intéressante pour des études spécifiques sur les caractéristiques des spectromètres à temps de vol [8]. En particulier la résolution en masse obtenue par la relation $\frac{\Delta M}{M}=\frac{1}{2} \frac{\Delta T}{T}(\Delta T$ étant la largeur à mi-hauteur d'un pic de temps de vol au temps $T$ dans le spectre) varie pour une molécule donnée avec la longueur de vol. Dans le cas d'un agré- 


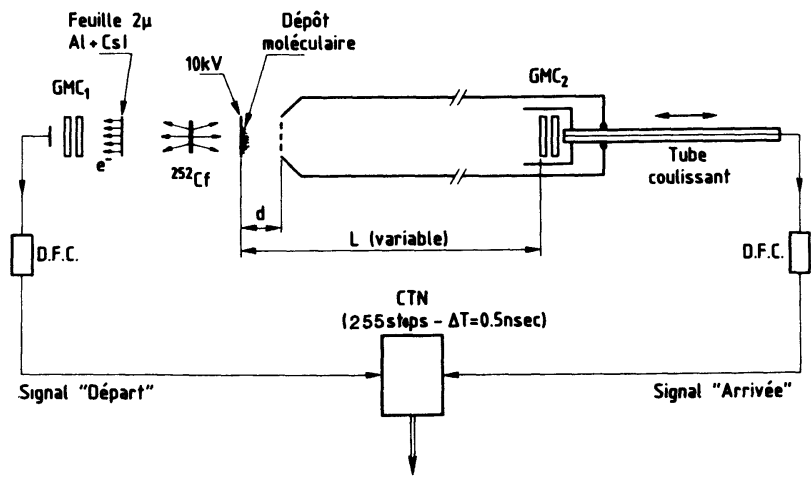

Vers système d'acquisition

Fig. 1. - Schéma de principe pour la mesure de spectres de masses de temps de vol : GMC : galettes microcanaux; DFC : discriminateur à fractions constantes; CTN : convertisseur temps numérique.

[Schematic diagram of the apparatus.]

gat de iodure de césium (ICs)Cs ${ }^{+}$, cette résolution varie linéairement de 300 à 1000 pour une longueur comprise entre 20 à $95 \mathrm{~cm}$. Il y a cependant une diminution de la transmission avec l'augmentation de longueur. Pour la mesure de la masse de grosses molécules nous utilisons généralement une distance de temps de vol de l'ordre de $30 \mathrm{~cm}$ et une distance d'accélération $(d)$ de $4 \mathrm{~mm}$. La haute tension qui accélère les ions créés par le passage du produit de fission à travers le dépôt moléculaire qui est appliqué entre l'échantillon et la grille ou les grilles extractrices maintenues à la masse, est aussi un autre paramètre. Dans les résultats montrés plus loin, le potentiel appliqué était de l'ordre de $16 \mathrm{kV}$. Une bien meilleure résolution en masse peut être obtenue à l'aide d'un miroir électrostatique [13] qui permet alors d'atteindre des valeurs $M / \Delta M$ de l'ordre de 5000 [14]. Le dispositif, grâce au miroir, est alors adapté à l'étude des décompositions en vol des ions métastables qui apportent des informations structurales sur la molécule qui se fragmente [15].

Pour positionner des échantillons entre l'extracteur et la source de ${ }^{252} \mathrm{Cf}$ un dispositif précis a été conçu au laboratoire. Dix échantillons peuvent être introduits par l'intermédiaire d'un sas dans l'enceinte sous vide. Une commande de mise en place de l'un ou l'autre de ces échantillons contrôlés par microprocesseur pré-programmé, permet à l'opérateur de choisir l'échantillon à analyser. Un schéma est présenté sur la figure 2 .

Les détecteurs d'ions sont des détecteurs microcanaux montés en chevron avec sortie d'anode à la haute tension $(\sim 3 \mathrm{kV})$, l'électronique associée est composée de 2 discriminateurs à fraction constante [16] et d'un convertisseur temps numérique (C.T.N.) également réalisé au laboratoire [17]. Le C.T.N. possède la particularité de classer pour un signal de départ jusqu’à 255 signaux « stop ", avec des

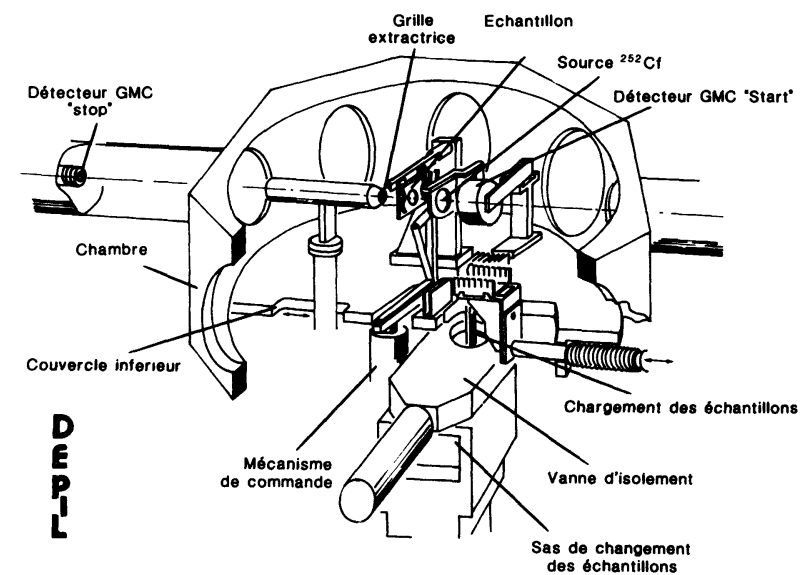

Fig. 2. - Schéma du centre de l'appareil DEPIL avec le mécanisme de passeur d'échantillons.

[Schematic diagram of the center of the DEPIL mass spectrometer.]

intervalles de temps minimum de 500 ps et un temps mort entre chaque "stop" de 30 ns. Les gammes de temps d'analyse sont comprises entre $16 \mu \mathrm{s}$ et $128 \mu \mathrm{s}$. L'acquisition de données se fait à l'aide d'un bloc mémoire de 16000 canaux "multispectral» Numelec. L'utilisation du spectromètre DEPIL en mode "métastable" avec le miroir électrostatique implique l'utilisation de moyens informatiques plus sophistiqués [15].

1.2 Préparation des échantillons. - L'échantillon est constitué d'un cadre supportant une feuille de mylar aluminisé (épaisseur mylar $+\mathrm{Al}$ de $1,5 \mu$ ) parfaitement plane. Sur la face aluminisée le dépôt de matière organique s'effectue par la technique d'électrospray à partir d'une solution plus ou moins concentrée du produit. Pour la plupart des composés organiques le solvant utilisé est un mélange méthanoléthanol-eau. Pour certaines molécules, protéines en particulier, l'acide acétique glacial ou acide trifluoracétique donnent de meilleurs résultats. Ces échantillons sont conservés sous vide ou sous atmosphère d'azote. Bien qu'une étude systématique n'ait pas été réalisée, il a été constaté une détérioration des échantillons de grosses protéines après quelques jours. Il faut noter à ce sujet que la méthode de mesure des masses n'est cependant pas destructive et le composé étudié peut dans la plupart des cas être récupéré après analyse. Il suffit en effet de $10^{3}$ à $10^{6}$ fragments de fission heurtant le dépôt organique (selon le composé à analyser) pour être en mesure de constituer un spectre. Ce nombre est très faible devant celui des molécules sur la surface. Au cours d'expériences sur des molécules de faible masse $(<1000) 80 \%$ de la quantité déposée ont pu être récupérés (3) après analyse. Enfin, plusieurs mesures peuvent être répétées sur le même échantillon. 
1.3 Calibration temps de vol-masse. - Des ions $\mathrm{H}^{+}$et $\mathrm{Na}^{+}$toujours émis de la surface par le fragment de fission qui la traverse permettent une calibration interne masse-temps de l'appareil. La masse de $\mathrm{Na}^{+}$ utilisée est 22,989 et celle de $\mathrm{H}^{+}$est légèrement inférieure à la valeur théorique $(1,00789)$ car il y a lieu de tenir compte d'une très faible vitesse initiale. La valeur déterminée empiriquement par $\mathrm{H}^{+}$est pour les tensions d'accélération utilisées de 1,0055 . Pour les ions négatifs, les masses utilisées pour la calibration sont $\mathrm{H}^{-}(1,0062)$ et $\mathrm{C}_{2} \mathrm{H}^{-}(25,007)$. Les constantes $(A$ et $B)$ de la relation $T=A \sqrt{M}+B$ sont déduites à l'aide de ces deux masses de calibration. La précision au niveau des masses 1000 est de $\pm 0,2$ unité de masse. Pour les masses élevées dépassant 10000 l'incertitude sur la détermination de la masse provient principalement de la soustraction du bruit de fond et de la détermination des bornes inférieures et supérieures du pic de temps de vol dont on calcule le centroïde. L'origine de ce bruit de fond observé dans les spectres provient essentiellement de la fragmentation de molécules désorbées qui se produit dans l'espace d'accélération.

La mesure de calibration est faite simultanément à la mesure des masses élevées. On utilise pour les masses faibles une sensibilité du C.T.N. de 0,5 ou $1 \mathrm{~ns} /$ canal, tandis que pour la zone de masses élevées une sensibilité de 16, 32 ou $64 \mathrm{~ns}$ /canal est généralement choisie par l'opérateur. Un spectre de calibration avec $1 \mathrm{~ns} /$ canal est présenté sur la figure 3. On y distingue parfaitement $\mathrm{H}^{+}$et $\mathrm{Na}^{+}$.

\section{Résultats sur des molécules de masses élevées.}

Afin d'évaluer les possibilités du spectromètre pour les mesures sur des masses élevées, des échantillons de protéines ont été préparées. Nous avons choisi les molécules suivantes :

Insuline bovine $\left({ }^{2}\right)$ masse calculée théorique : 5733,5 Insuline porcine $\left({ }^{2}\right)$ masse calculée théorique : 5 777,6.

Ces molécules ont déjà été observées en spectrométrie de masse $[11,18,19]$ et bien que n'étant pas des composés facilement mesurables, ils constituent cependant des standards pour estimer la potentialité d'un instrument pour les masses élevées. Signalons qu'un spectromètre à temps de vol permet d'explorer une gamme de masse complète (par exemple 1 à 30 000) au cours d'une même mesure. Ceci n'est pas possible avec un appareil à secteur magnétique.

Sur les figures $4 \mathrm{a}$ et $\mathrm{b}$ sont présentés les spectres de masse de l'insuline bovine pour la région de l'ion moléculaire. La figure 4a représente le spectre brut obtenu avec $32 \mathrm{~ns} /$ canal sur une période de mesure de $10 \mathrm{~h}$. Après quelques minutes d'accumulation le pic moléculaire est déjà nettement visible. La figure $4 \mathrm{~b}$ est le même spectre après soustraction du bruit de fond effectué à l'ordinateur. La valeur de la masse

(2) Provenance : SIGMA.

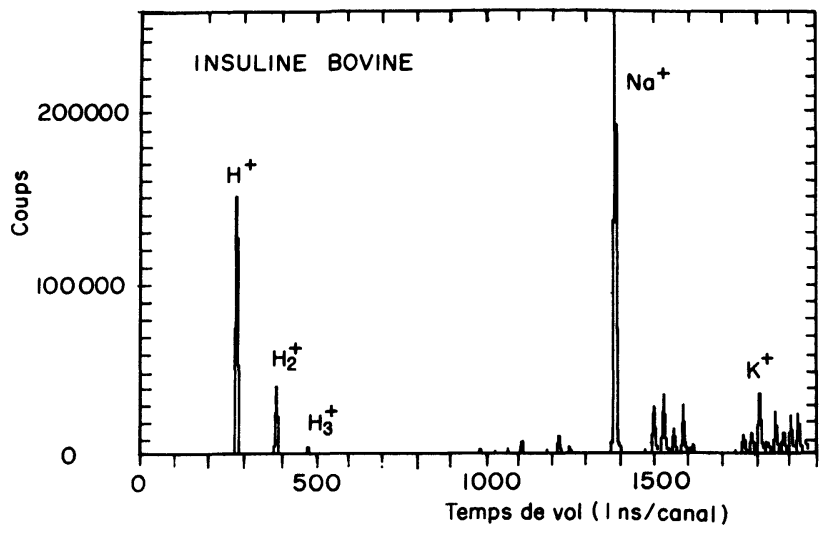

Fig. 3. - Spectre de temps de vol pour la calibration tempsmasse $(T=A m+B)$ présentant les pics de calibration $\mathrm{H}^{+}$ et $\mathrm{Na}^{+}$mesurés simultanément avec le spectre total de l'insuline bovine (Fig. 4). La partie du spectre pour les temps inférieurs à 4000 ns est systématiquement enregistrée avec une définition de $1 \mathrm{~ns} / \mathrm{canal}$.

[Time-of-flight mass spectrum showing the peaks $\mathrm{H}^{+}$and $\mathrm{Na}^{+}$used for mass calibration. The part of the spectrum for $T<4000 \mathrm{~ns}$ is always recorded with $1 \mathrm{~ns} /$ channel.]
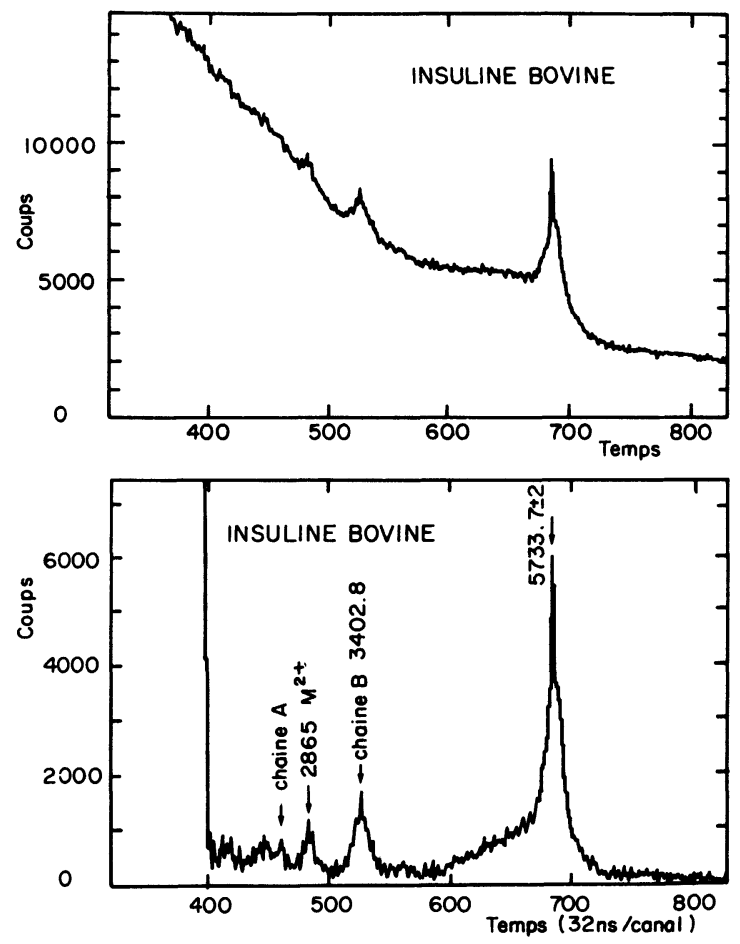

Fig. 4. - a) Spectre de masse mesuré avec une définition de $32 \mathrm{~ns} / c a n a l$ d'une molécule d'insuline bovine (durée comptage $10 \mathrm{~h}$ ).

b) Le même spectre après soustraction du bruit de fond.

[a) Time-of-flight mass spectrum obtained with time bins of $32 \mathrm{~ns} /$ channel for a molecule of bovine insuline. b) Same spectrum after background subtraction.] 
calculée d'après le centroïde du pic (au-dessus de la mi-hauteur) est 5733 en bon accord avec la masse théorique moyenne de 5733,5 calculée en tenant compte des abondances isotopiques. Le très bon accord obtenu pour cette mesure est cependant fortuit car une incertitude de l'ordre de $\pm 4 \mathrm{u}$ a été estimée pour la détermination des masses d'insuline.

Sur le spectre de la figure $4 \mathrm{~b}$, l'ion moléculaire doublement chargé $\mathrm{M}^{2+}$ ainsi que le fragment (chaîne B de l'insuline) apparaissent bien distinctement. Le fragment-chaîne A est peu visible.

La figure 5 montre la région du pic moléculaire avec une sensibilité plus grande de $4 \mathrm{~ns} / \mathrm{canal}$. L'on y distingue plusieurs composantes à $5729,7,5740,8$ et 5 788. La présence de ces composantes peut provenir d'un manque de pureté du produit initial ou de modifications moléculaires intervenant au cours de la préparation de l'échantillon. Cette analyse montre que la détermination de masse avec une faible intensité requiert certaines précautions.

La figure 6 montre une mesure pour un autre type de molécule d'insuline : l'insuline porcine de masse théorique 5777,6 . Le spectre obtenu après soustraction du bruit de fond montre l'ion moléculaire mesuré à $m=5777 \pm 3$. La mesure d'une durée de quelques heures a été réalisée avec une sensibilité de $64 \mathrm{~ns} / \mathrm{canal}$.

Enfin, des poids moléculaires nettement plus élevés ont été mesurés avec une très grosse protéine : la " lyzozyme " $\left({ }^{3}\right)$ comprenant de l'ordre de 129 acides aminés. Une dizaine de microgrammes de lyzozyme ont été dissous dans l'eau. L'électrospray a été effectué à partir de cette solution additionnées d'isopropanol.

Le spectre de masse après soustraction du bruit de fond est montré sur la figure 7. La mesure a été faite avec une résolution temps de $64 \mathrm{~ns} / \mathrm{canal}$. Compte tenu de la statistique assez faible, de la soustraction du bruit de fond et de la définition des bornes des pics,

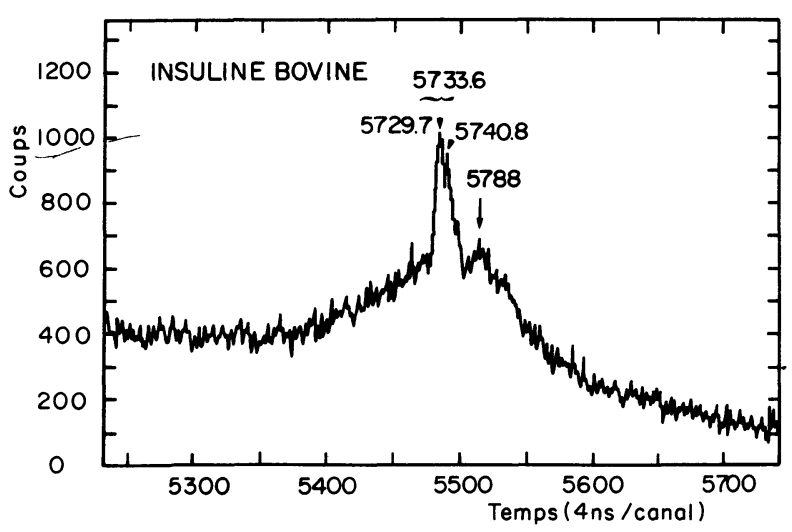

Fig. 5. - Région du pic moléculaire de la figure 4 présentée avec une définition de 4 ns/canal.

[Molecular peak region of figure 4 shown with $4 \mathrm{~ns} /$ channel.]

$\left({ }^{3}\right)$ Provenance : Boehringer-Mannheim (RFA).

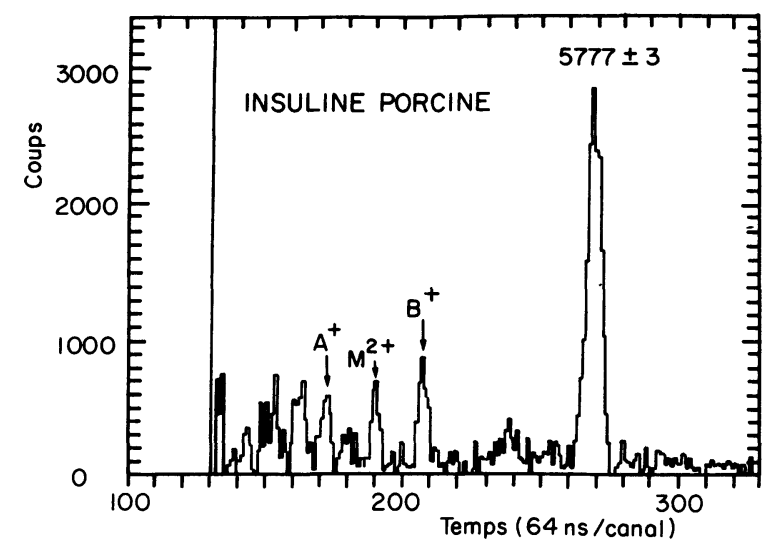

Fig. 6. - Spectre de masse après soustraction de bruit de fond de la molécule d'insuline porcine.

[Mass spectrum of the molecule porcine insuline after background subtraction.]

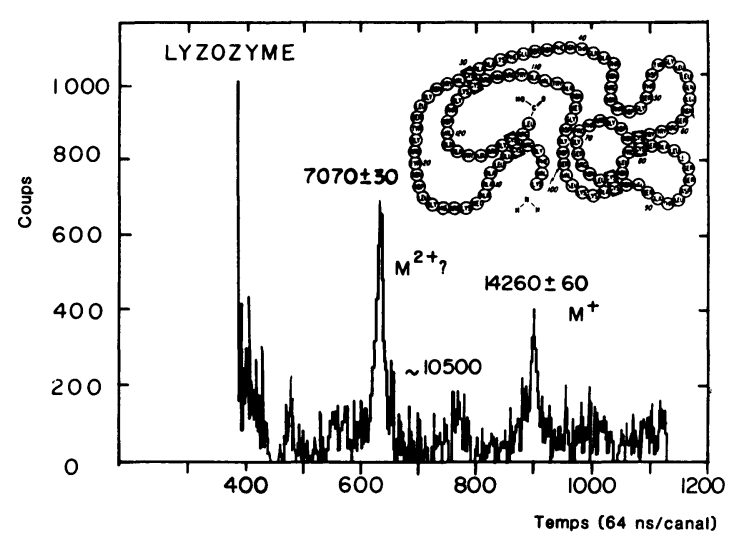

Fig. 7. - Spectre de masse de la protéine lyzozyme comprenant 129 acides aminés.

[Mass spectrum of the protein lyzozyme (129 aminoacids).]

nous avons indiqué les valeurs minimales et maximales pour les masses des deux pics cependant nettement visibles dans ce spectre. Une purification du produit et une optimisation des calibrations de masses devraient permettre d'atteindre une incertitude inférieure à 10 unités de masse. Les valeurs de masses estimées par différents auteurs (Refs. [20-22]) à l'aide de méthodes biophysiques s'étalent entre 14100 et 14600 . Il est probable que les deux pics dans le spectre correspondent aux ions $\mathbf{M}^{2+}$ et $\mathbf{M}^{+}$.

\section{Conclusion.}

On a montré que le spectromètre de masse par temps de vol DEPIL réalisé à l'Institut de Physique Nucléaire était, en dehors de ses autres possibilités, adapté à la mesure de masses de très grosses molécules. Un résultat original sur la protéine lyzozyme a été obtenu au cours de ce travail qui s'inscrit dans un programme 
de recherche sur la mesure et la détection de très hautes masses. Les instruments temps de vol apparaissent dans ce contexte bien adaptés, car ils peuvent couvrir une large gamme de masses et sont moins sensibles yue les autres instruments aux fragmentations des molécules. En effet, les ions moléculaires acquièrent, après accélération, une certaine vitesse qui est con iervée par les fragments s'il y a cassure en vol. Dans ce cas la détection d'un fragment donne la même information que la masse totale. Les problèmes fondamentaux de création des ions sont évidemment très liés à ceux de leur détection ultérieure et nécessitent donc parallèlement des études spécifiques. Dans le domaine d'énergies de plusieurs dizaines de $\mathrm{MeV}$, ces études requièrent l'utilisation d'accélérateurs d'ions lourds, mais appellent aussi la comparaison avec d'autres méthodes d'ionisation-désorption (laser et ion de quelques $\mathrm{keV}$ notamment). Ces phénomènes physico-chimiques d'émission secondaire d'ions polyatomiques ou moléculaires ne sont pas bien connus; ils mettent en jeu des phénomènes liés aux surfaces, à la perte et aux transferts d'énergie dans les interactions ion-matière. Il $\mathrm{y}$ a des paramètres physiques contrôlables dans la voie d'entrée (nature, vitesse, états de charge des ions incidents...) et dans la voie de sortie (types d'ions atomiques ou moléculaires émis, durée de vie, rendement d'émission...).

Les ions multichargés de plusieurs dizaines de $\mathrm{MeV}$ apparaissent très efficaces pour la désorption de très grosses entités. La compréhension des processus au niveau fondamental est certainement très utile pour l'exploitation des méthodes au niveau analytique. Des interprétations théoriques des mécanismes mis en jeu ont été avancées par divers auteurs [23-25] et ont été comparées à divers résultats obtenus au laboratoire sur les fonctions d'excitation de rendement d'émission secondaire [26]. Il n'y a pas pour l'instant d'explications complètement satisfaisantes. Des résultats récents ont de plus montré l'importance de la nature du support sur lequel les dépôts organiques solides sont effectués. Il apparaît que les énergies de liaison des molécules à certain substrat [27-28] peuvent être abaissées et que l'énergie interne transmise lors de la désorption est plus faible, réduisant par conséquent le nombre de fragmentations en vol.

Il est clair que pour les protéines de masses élevées un soin particulier doit être apporté à leur purification puis à la préparation des échantillons. La spectrométrie de masse peut ensuite apporter des informations fondamentales.

\section{Remerciements.}

Ce travail a pu être initié et réalisé grâce à la compétence des services Etude, Mécanique, Electronique de l'I.P.N. Nous remercions vivement toutes les personnes qui ont contribué à cette réalisation. Nous remercions aussi les personnes qui ont permis la mise en forme de ce travail et particulièrement $E$. Davanture. Nous apprécions la participation du Centre de Génétique Moléculaire du CNRS de Gif qui nous a fourni quelques produits.

\section{References}

[1] Della Negra, S., Deprun, C., Gauvin, H., Husson, J. P., JungClas, H. and Le BeyeC, Y., Nucl. Inst. Meth. 156 (1978) 355.

[2] Della Negra, S., Gauvin, H., Jacquet, D. and Le BEYEC, Y., Z. für Physik A 307 (1982) 305.

[3] Le Beyec, Y., Della Negra, S., Deprun, C., Vigny, P. and Ginot, Y. M., Revue Phys. Appl. 15 (1980) 1631.

[4] Posthumus, A. M., Kistmaker, P. G., Meuzelaar, H. L. C., Ten Noeuver de Brau, M. C., Anal. Chem. 50 (1978) 985.

[5] TABet, J. C. and CotTer, R., Anal. Chem. 56 (1984) 1662.

[6] Steffens, P., Niehuis, E., Friese, T. and BenninghoVEN, A., in Ion Formation from Organics Solids, Springer series in Chemical Physics (Springer, Berlin) 1983, vol. 25, p. 111.

[7] Standing, K. G., Beavis, R., Bolbach, G., Ens, W., Main, D. E. and Schueler, B., Proc. of the Conf. on SIMS V, Washington D.C., oct. 1985.

[8] Torgerson, D. F., Skowronski, R. P. and MacfarLANE, R. D., Biochem. Biophys. Mass. Commun 60 (1974) 616.

[9] Della Negra, S. and Le Beyec, Y., Nucl. Sci. Appl. 1 (1983) 569.
[10] Macfarlane, R. D., Analyt. Chem. 55A (1983) 1247.

[11] Sundquist, B., Kamensky, I., Hakansson, P., KJellBerg, J., Sulehpour, M., Widdigasekera, S., Fohlman, J., Peterson, P. A., Roepstorff, P., Biomech. Mass. Spectr. 11 (1984) 242.

[12] Sundquist, B., RoepstorfF, P. et al., Sciences 226 (1984) 696-698.

[13] Della Negra, S. and Le BeyeC, Y., Int. J. Mass. Spectrom. Ion Phys. 61 (1984) 21.

[14] Della Negra, S., Le Beyec, Y., Standing, K., résultats non publiés.

[15] Della Negra, S. and Le Beyec, Y., Anal. Chem. 50 (1985) 2035.

[16] Pouthas, J. and Engrand, M., Nucl. Instrum. Method 161 (1979) 361.

[17] Festa, E., Sellem, R., Nucl. Instrum. Method 188 (1981) 99.

Festa, E., Sellem, R., Tassangot, L., Nucl. Instrum. Meth. Phys. Res. A 234 (1985) 305.

[18] Barber, M., Bordoli, R. S., Elliot, G. J., Horoch, N. J., Green, B. N., Biochem. Biophys. Res. Commun. 110 (1983) 753.

[19] Cotter, R. J., Larsen, B. S., Heller, D. N., Campana, J. E. and Fenselan, C., Anal. Chem. 57 (1985) 1479. 
[20] MAHLher, H. R., Biological Chemistry (Harper International Edition) 1969.

[21] Canfield, R. E. and Liu, A. K., J. Biol. Chem. 240 (1965) 1997-2002.

[22] Jolles, J., Jauregui-Adell, J. and Jolles, P., Biochem. Biophys. Acta 71 (1963) 488.

[23] Hedin, A., Häkansson, P., Sundquist, B., JohnSON, R. E., Phys. Rev. 31, 4 (1985) 1780.

[24] Watson, C. C. and Tombrello, T. A., Rad. Eff. 89 (1985) 263.
[25] Kammer, H. F., Thesis, T. H. Darmstadt (1985), to be published.

[26] Becker, O., Della Negra, S., Le Beyec, Y. and WIEN, K., IPNO-DRE-85-21, Nucl. Instrum. Meth. (in press).

[27] Sundquist, B., Hedin, A., HäKansson, P., JohnSon, G., Salehpour, M., Säve, G., RoepstorfF, P., Proc. of the IFOS III Meeting, Munster, 1985, (edited by Springer).

[28] Alai, M., Demirev, P., Fenselau, C. and Cotter, R., in press Anal. Chem. (1986). 